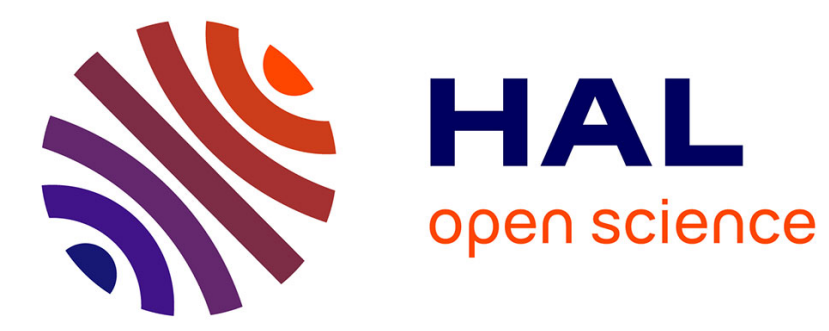

\title{
Periodic Thermodynamics of Isolated Quantum Systems
}

Achilleas Lazarides, Arnab Das, Roderich Moessner

\section{To cite this version:}

Achilleas Lazarides, Arnab Das, Roderich Moessner. Periodic Thermodynamics of Isolated Quantum Systems. Physical Review Letters, 2014, 112, pp.150401. 10.1103/PhysRevLett.112.150401 . hal03019409

\section{HAL Id: hal-03019409 \\ https://hal.science/hal-03019409}

Submitted on 23 Nov 2020

HAL is a multi-disciplinary open access archive for the deposit and dissemination of scientific research documents, whether they are published or not. The documents may come from teaching and research institutions in France or abroad, or from public or private research centers.
L'archive ouverte pluridisciplinaire $\mathbf{H A L}$, est destinée au dépôt et à la diffusion de documents scientifiques de niveau recherche, publiés ou non, émanant des établissements d'enseignement et de recherche français ou étrangers, des laboratoires publics ou privés. 


\title{
Periodic Thermodynamics of Isolated Quantum Systems
}

\author{
Achilleas Lazarides, ${ }^{1}$ Arnab Das, ${ }^{1,2}$ and Roderich Moessner ${ }^{1}$ \\ ${ }^{1}$ Max-Planck-Institut für Physik komplexer Systeme, 01187 Dresden, Germany \\ ${ }^{2}$ Theoretical Physics Department, Indian Association for the Cultivation of Science, Kolkata 700032, India
}

(Received 24 January 2014; published 16 April 2014)

\begin{abstract}
The nature of the behavior of an isolated many-body quantum system periodically driven in time has been an open question since the beginning of quantum mechanics. After an initial transient period, such a system is known to synchronize with the driving; in contrast to the nondriven case, no fundamental principle has been proposed for constructing the resulting nonequilibrium state. Here, we analytically show that, for a class of integrable systems, the relevant ensemble is constructed by maximizing an appropriately defined entropy subject to constraints, which we explicitly identify. This result constitutes a generalization of the concepts of equilibrium statistical mechanics to a class of far-from-equilibrium systems, up to now mainly accessible using ad hoc methods.
\end{abstract}

DOI: 10.1103/PhysRevLett.112.150401

PACS numbers: 05.30.Ch, 37.10.Jk, 67.85.-d

There has recently been significant progress in our understanding of statistical mechanics based on the twin concepts of equilibration, the approach of a large, closed system's state to some steady state [1-8], as well as of thermalization, when this steady state depends only upon a small number of quantities. Starting from ideas due to Jaynes [9,10], Srednicki and Deutsch [1,2], and Popescu et al. [11], both integrable and nonintegrable closed, nondriven many-body systems have, thus, been shown to thermalize $[3,5,8]$.

On the other hand, the study of periodically driven systems has also had a long history. Following early foundational work by Shirley [12] and Sambe [13], substantial theoretical and experimental progress has recently been made [14-22].

Here, we combine ideas from the two areas to extend the concept of thermalization to the out-of-equilibrium case of periodically driven systems. By devising a mapping of the system to a set of effectively nondriven systems, we show that a periodically driven system asymptotically approaches a time-periodic steady state at long times (see, e.g., Ref. [23] and the Supplemental Material [24]). Specializing to a large class of integrable systems, we analytically show that Jaynes's entropy maximization principle $[9,10]$ gives a statistical mechanical description of the long-time, synchronized dynamics for infinite systems and study the approach to this equilibrium state as a function of both the system size and time. Finally, we explain how our proposed setup is achievable with current experimental techniques.

Synchronization.-The starting point for our analysis is the synchronization of the system with the driving, which may be seen as follows.

Consider a time-periodic Hamiltonian $\hat{H}(t)=\hat{H}(t+T)$ and denote the time evolution operator over a period starting from time $0 \leq \epsilon<T$ by $\hat{U}(\epsilon, \epsilon+T)$. Taking $\hbar=1$, we define an effective Hamiltonian $\hat{H}_{\text {eff }}$ via

$$
\exp \left[-i \hat{H}_{\mathrm{eff}} T\right]=\hat{U}(0, T) .
$$

$\hat{H}_{\text {eff }}$ is a time-independent effective Hamiltonian that takes an initial state at $t=0$ to the same final state at $t=T$ as the real time-dependent Hamiltonian $\hat{H}(t)$.

We concentrate on "stroboscopic" observations, that is, observations at discrete points of time separated by a period $t_{n}=\epsilon+n T$ for a given $\epsilon$. The expectation value of an arbitrary time-independent operator $\hat{O}$ at time $t$, $\mathcal{O}(t)=\langle\psi(t)|\hat{O}| \psi(t)\rangle$, is

$$
\mathcal{O}\left(t_{n}\right)=<\psi(0)\left|e^{i \hat{H}_{\text {eff }} n T} \hat{O}^{(e)} e^{-i \hat{H}_{\text {eff }} n T}\right| \psi(0)>
$$

where $\hat{\mathcal{O}}^{(\epsilon)}=\hat{U}^{\dagger}(0, \epsilon) \hat{\mathcal{O}} \hat{U}(0, \epsilon)$. We have, thus, recast the time evolution into evolution under a time-independent Hamiltonian, at the price of introducing a set of new operators $\hat{\mathcal{O}}^{(\epsilon)}$.

By analogy to a static quench $[4,7]$ (see the Supplemental Material [24] for a discussion of the necessary conditions), one can show that each series $\left\{\mathcal{O}\left(t_{n}\right) ; n=\right.$ $0,1,2, \ldots\}$ converges to a fixed value. This immediately implies that the long-time behavior of the system is periodic in time, i.e., synchronized.

Construction of the periodic ensemble.-We now come to the main part of our work where we show that Jaynes's idea of entropy maximization $[4,5,9,10]$ remains valid away from equilibrium for this class of models. In order to demonstrate that this is correct, we restrict ourselves to a class of tractable integrable Hamiltonians. For infinite systems, we show analytically that this ensemble correctly reproduces all correlation functions. For finite systems, we study the approach to the thermodynamic limit in a spatially inhomogeneous system of hard-core bosons (HCBs).

The Hamiltonians we consider are of the form

$$
\hat{H}(t)=\sum_{i}\left[\hat{a}_{i}^{\dagger} \mathcal{M}_{i, j}(t) \hat{a}_{j}+\hat{a}_{i}^{\dagger} \mathcal{N}_{i, j}(t) \hat{a}_{j}^{\dagger}+\text { H.c. }\right],
$$


with the $\hat{a}_{i}$ fermionic or bosonic operators, $\left[a_{i}, a_{j}^{\dagger}\right]_{ \pm}=\delta_{i, j}$, and $\mathcal{M}, \mathcal{N}$ are complex matrices. In cases of interest, the nonlinear, nonlocal transformation that brings the physical Hamiltonian to this form maps local observables to highly nonlocal, nonlinear functions of the $\hat{a}$ operators.

For Hamiltonians bilinear in the operators $\hat{a}, \hat{H}_{\text {eff }}$ are bilinear and may, therefore, be brought to the form

$$
\hat{H}_{\text {eff }}=\sum_{p=1}^{L} \omega_{p} \tilde{a}_{p}^{\dagger} \tilde{a}_{p}
$$

by a unitary transformation ( $L$ is the system size). The operators $\hat{\mathcal{I}}_{p}(t):=\hat{U}(0, t) \tilde{a}_{p}^{\dagger} \tilde{a}_{p} \hat{U}^{\dagger}(0, t)$ (of which there are $L)$ correspond to conserved quantities $\left\langle\psi(t)\left|\hat{\mathcal{I}}_{p}(t)\right| \psi(t)\right\rangle=\left\langle\psi(0)\left|\hat{\mathcal{I}}_{p}(0)\right| \psi(0)\right\rangle$ for all $t$ and are temporally periodic.

We now describe how to obtain the statistical ensemble describing the long-time behavior of this system after a number of periods have elapsed. Given the set $\left\{\hat{\mathcal{I}}_{p}(t)\right\}$, we construct the most general distribution maximizing Shannon's entropy in the space of periodic operators, subject to the constraints given by the conservation laws. The resulting "periodic Gibbs ensemble" (PGE) density operator is

$$
\hat{\rho}_{\mathrm{PGE}}(t)=\mathcal{Z}^{-1} \exp \left(-\sum_{p} \lambda_{p} \hat{\mathcal{I}}_{p}(t)\right)
$$

with the $\lambda_{p}$ value fixed by requiring that $\left\langle\psi(0)\left|\hat{\mathcal{I}}_{p}(0)\right| \psi(0)\right\rangle=\operatorname{tr}\left[\hat{\rho}_{\mathrm{PGE}}(0) \hat{\mathcal{I}}_{p}(0)\right] \quad$ and $\quad \mathcal{Z}=$ $\operatorname{tr}\left[\exp \left(-\sum_{p} \lambda_{p} I_{p}\right)\right]$, a (time-independent) normalization factor. Operator $\hat{\rho}_{\mathrm{PGE}}(t)$ has the following two properties: First, it correctly gives the conserved quantities: $\operatorname{tr}\left[\tilde{a}_{p}^{\dagger} \tilde{a}_{q} \hat{\rho}_{\mathrm{PGE}}(t)\right]=\delta_{p, q}\left\langle\psi(t)\left|\hat{\mathcal{I}}_{p}(t)\right| \psi(t)\right\rangle$. Second, since the $\hat{\mathcal{I}}_{p}$ are periodic in time, it is itself manifestly periodic with time: $\hat{\rho}_{\mathrm{PGE}}(t)=\hat{\rho}_{\mathrm{PGE}}(t+T)$.

Finally, we can analytically show that the PGE density matrix exactly reproduces all correlation functions in the thermodynamic limit; this somewhat lengthy but ultimately elementary calculation is described in the Supplemental Material [24]. This constitutes our central conceptual result.

Application to finite systems: numerical results. - Let us now supplement the above exact and general results using numerical simulations for specific, finite systems. While the proof for the correctness of the PGE is strictly applicable only in the thermodynamic limit, we shall see that the deviation of finite systems from the PGE result rapidly decreases with system size.

A number of different physical systems may be mapped to Eq. (3) (see Supplemental Material [24]). Here, we present numerical results for the experimentally relevant case of HCBs subject to a simple potential, the Hamiltonian for which reads

$$
\hat{H}_{b}(t)=-\frac{1}{2} \sum_{i} J_{i}(t) \hat{b}_{i}^{\dagger} \hat{b}_{i+1}+\text { H.c. }+\sum_{i} V_{i}(t) \hat{b}_{i}^{\dagger} \hat{b}_{i}
$$

with the $\hat{b}_{i}$ HCBs. The HCBs are described by operators $\hat{b}$ obeying bosonic commutation relations $\left[\hat{b}_{i}, \hat{b}_{j}^{\dagger}\right]=\delta_{i, j}$, with the additional hard-core condition $\hat{b}_{i}^{2}=0$. A JordanWigner transformation $\hat{b}_{i}=\hat{a}_{i} \prod_{j<i}(-1)^{\hat{n}_{j}}$ with $\hat{n}_{j}=$ $\hat{b}_{j}^{\dagger} \hat{b}_{j}=\hat{a}_{j}^{\dagger} \hat{a}_{j}$ maps $\hat{H}_{b}(t)$ to Eq. (3) with $\mathcal{M}_{i, j}(t)=$ $-\frac{1}{2} J_{i}(t)\left(\delta_{i+1, j}+\delta_{i-1, j}\right)+\delta_{i, j} V_{i}(t), \mathcal{N}_{i, j}=0$ and fermionic commutation relations for the $\hat{a}$.

Here, we focus on a time-dependent superlattice potential superposed on a quadratic potential, $V_{i}(t)=$ $\frac{1}{2}\left[(i-L / 2) / \ell_{\mathrm{HO}}\right]^{2}+\Delta(-1)^{i} \cos (\omega t)$ and a time-dependent hopping amplitude $J_{i}(t)=J+\delta J \cos (\omega t)$ with $\omega=2 \pi / T$. The protocol we use is to prepare the system in the ground state in the presence of a harmonic potential $V_{i}^{(0)}=\frac{1}{2}\left[(i-L / 2) / \ell_{\mathrm{HO}}\right]^{2}$, fixing $\ell_{\mathrm{HO}}=N$. This allows us to take the thermodynamic limit, since for large total number of particles the dimensionless parameter [25] $\tilde{\rho}=N_{b} / \ell_{\text {HO }}$ plays a role analogous to the density in the uniform limit. Results with different system sizes but constant $\tilde{\rho}$ are therefore comparable.

At time $t=0$, the driving is switched on so that the total Hamiltonian is $\hat{H}_{b}(t)=-\frac{1}{2} J \sum_{i} \hat{b}_{i}^{\dagger} \hat{b}_{i+1}+$ H.c. + $\sum_{i} V_{i}(t) \hat{b}_{i}^{\dagger} \hat{b}_{i}$ with $V_{i}(t)=V_{i}^{(0)}+\Delta(-1)^{i} \cos (2 \pi t / T)$.

Concentrating on the experimentally accessible momentum distribution of the bosons $\hat{n}^{(b)}(k)=L^{-1} \sum_{i, j} \hat{b}_{i}^{\dagger} \hat{b}_{j} \times$ $\exp \left[-2 \pi k(i-j) L^{-1}\right]$, we use the numerical method used in, among others, Ref. [26]; it consists of solving the fermionic time-dependent problem and, at the end, inverting the Jordan-Wigner transformation [27]. We begin by demonstrating a number of possible periodic states, corresponding to different parameters of the model. The leftmost panel of Fig. 1 shows snapshots of the PGE momentum distribution $\operatorname{tr}\left[\hat{\rho}_{\mathrm{PGE}} \hat{n}^{(b)}(k)\right]$ at the beginning of each period $(\epsilon=0)$ for different parameter values. We emphasise that, away from the high-frequency regime, the corresponding time-averaged Hamiltonian $[16,28]$ is not an appropriate description. As a striking example, the black line shows a momentum distribution with peaks at the edges of the Brillouin zone. Concentrating now on the parameters corresponding to the cyan line, the central panel shows the time evolution of the momentum distribution over an entire period. Note that the system evolves through states in which the momentum is peaked at different locations of the Brillouin zone. Finally, the rightmost panel shows three snapshots of the density distribution of the same system at times indicated by the colored lines in the central panel. The high spatial frequency oscillations and the peaking of the density at the edges is also very different from what would be obtained had the system been well described by a time-averaged Hamiltonian, since the time-averaged potential (shown in black) is smooth and its potential is highest at the edges.

We next discuss the approach to the long-time periodic state as a function of time and system sizes. After showing that the stroboscopic values of observables approach, then oscillate around, a constant value for each $\epsilon$, we proceed 


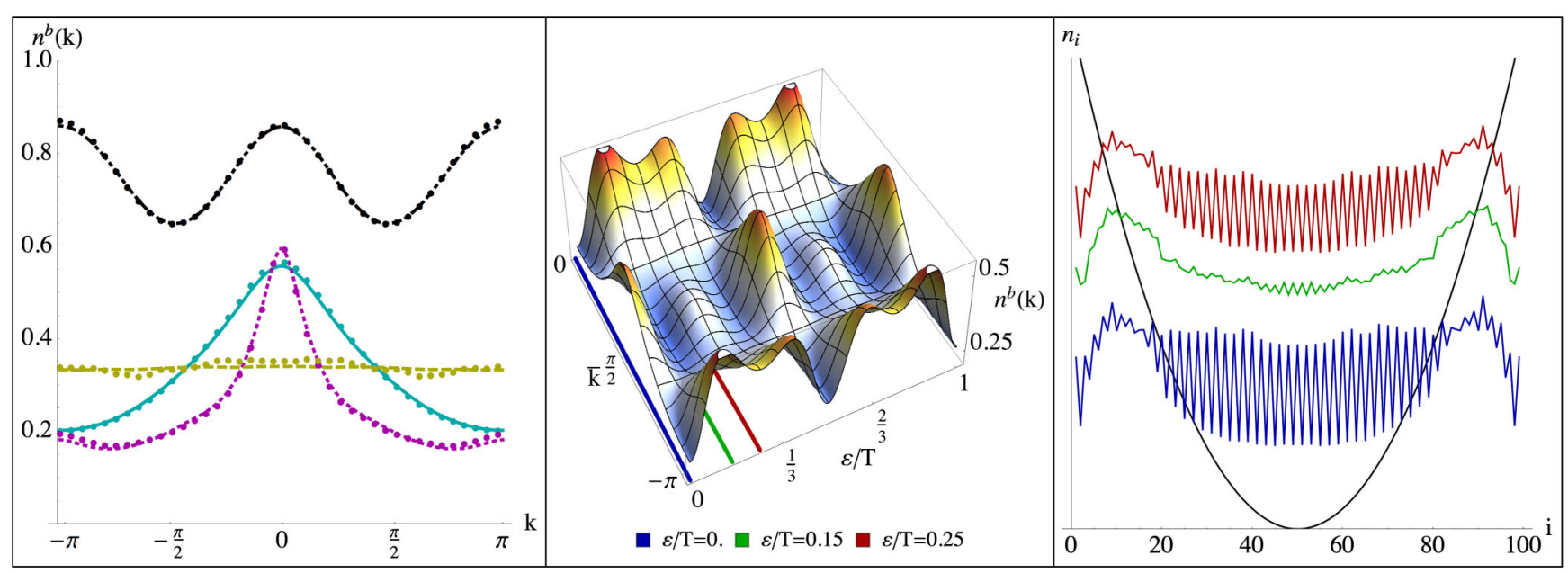

FIG. 1 (color online). Characterization of the synchronized steady state. Left: Stroboscopic momentum distribution, $\hat{n}(k)=L^{-1} \sum_{i, j} \hat{b}_{i}^{\dagger} \hat{b}_{j} \exp \left[-2 \pi i k(i-j) L^{-1}\right]$, demonstrating the wide range of behavior that occurs for varying parameters. The points correspond to snapshots of the dynamical evolution at late times $(t=490 T)$ for $L=200$, while the continuous lines correspond to the PGE prediction. From top to bottom at the extreme left end of the plot, the amplitudes of the superlattice potential, frequency, and filling factor $(\Delta, \delta J, \omega, \nu)$ are $(0.6,0.5,1.6,3 / 4)$ (black, dot-dashed), $(4,0.5,1.5,1 / 3)$ (yellow, dashed), $(4,0.75,2,1 / 3)($ cyan, full), $(0.6,0.5$, $2,1 / 4)$ (magenta, dotted), and $\epsilon=0$. The next two panels correspond to the parameters for the cyan full line. Center: Expectation value of the momentum distribution $\hat{n}(k)$ of the bosons during a single period in the synchronized state as a function of the time in the period $\epsilon$. The three lines on the time-momentum plane indicate the times $\epsilon / T=0,0.15,0.25$ for which density distributions are shown in the rightmost panel. The momentum distribution undergoes qualitative changes: at some points of the period, it has a single maximum at $k=0$ while at others it acquires double maxima at the edges of the Brillouin zone. Right: Each trace shows the expectation value of the density of the bosons $\hat{n}_{i}^{b}=\hat{b}_{i}^{\dagger} \hat{b}_{i}$ at the time indicated in the middle panel by the line of the same color, for a lattice size $L=100$ and offset for better visibility. The black line indicates the time average of the applied potential; the density peaks at the edges despite the potential being highest there indicating a strongly nonequilibrium situation.

to demonstrate that both this average value and the relative magnitude of the oscillations away from it decay to zero with increasing system size, in agreement with our analytical results for infinite systems. The approach is rapid: within a few periods, the system is practically thermalized.

The main plot of Fig. 2 shows the stroboscopic approach to the PGE state of the full bosonic momentum distribution, $\hat{n}^{(b)}(k, m T)$, for the parameters corresponding to the black line in Fig. 1. The entire momentum distribution approaches, then oscillates around, a period-independent result. The inset focuses on the component $\hat{n}^{(b)}(k=\pi / 2)$, showing the stroboscopic time evolution of its difference from the value predicted by the PGE as a function of period, showing the oscillations about the equilibrium value shown by the heavy blue lines.

We now quantitatively study the approach to the PGE limit as system size is increased. In Fig. 3, we plot the average of the distance of the dynamical momentum distribution from its PGE value over a number of periods, $\bar{d}=(L N)^{-1} \sum_{m=n}^{n+N} \sum_{k}\left|\hat{n}^{(b)}(k, m T)-\hat{n}_{\mathrm{PGE}}(k)\right|$, as a function of the inverse system size $1 / L$. These plots are for large $n=40 L$ and $N=20 L$ in order to to allow plenty of time for equilibration. From Fig. 3, we conclude that the average of the momentum distribution approaches the PGE result, while fluctuations away from it on average become smaller with increasing system size: as $L \rightarrow \infty$, the momentum distribution rapidly approaches the PGE periodic steady state.
In conclusion, we have shown that the real dynamics rapidly approaches the thermodynamic-limit and long-time results for relatively small systems and short times.

Experiments.-We now turn to the question of the experimental implementation of the specific system we have studied. To realize our proposal, three ingredients are required: A superlattice potential, periodic modulation, and HCBs.

Experiments using a superlattice potential are already available [29], while periodic modulation of the lattice depth $[18,30]$ is a standard technique. In particular, periodically driving a superlattice potential is described in Ref. [21]. Finally, the HCB regime may be achieved via confinement-induced resonance, which involves manipulating the radial harmonic potential strength $[31,32]$.

The example we have studied above is, therefore, accessible with current experimental techniques.

Conclusions and outlook.-For a large class of integrable periodically driven systems, we have shown that a periodic steady state is attained at long times. To describe this state, we have constructed a periodic version of the generalized Gibbs ensemble (GGE) [5], commonly introduced in connection with quenches in integrable models. We have provided an analytical demonstration that it exactly reproduces the periodic steady state in the thermodynamic limit. We also provide numerical evidence of rapid convergence (i) to the thermodynamic-limit 


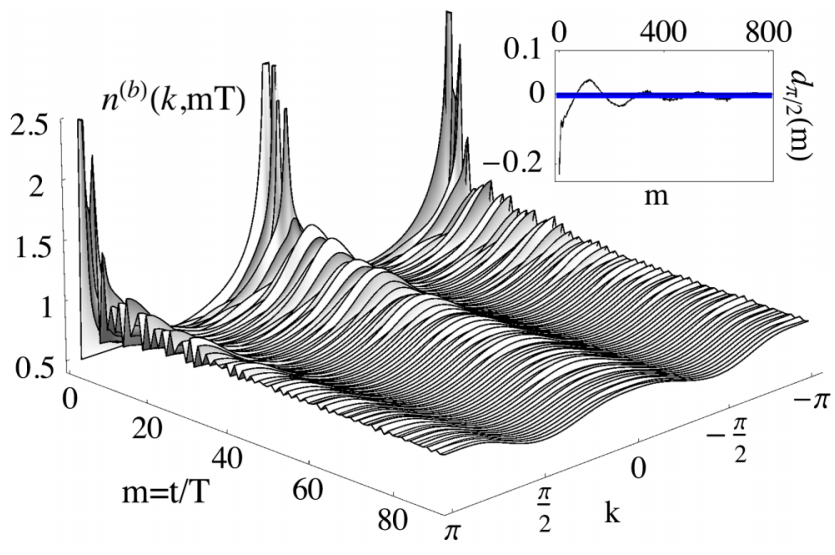

FIG. 2 (color online). Main plot: Stroboscopic approach to equilibrium with time for the full momentum distribution of the bosons $\hat{n}^{(b)}$, corresponding to the heavy black line in Fig. 1 and for a system size $L=200$ sites. Note the brief initial transient period, followed by small oscillations around a well-defined limit. Inset: Same as the main plot, but for a single component of the momentum distribution. In this plot, $d_{\pi / 2}(m)=$ $\left[\hat{n}^{(b)}(k=\pi / 2, m T)-\hat{n}_{\mathrm{PGE}}^{(b)}(k=\pi / 2)\right] / \hat{n}_{\mathrm{PGE}}^{(b)}(k=\pi / 2)$ measures the deviation of the actual value from the prediction of the PGE. The heavy blue lines show the average of the deviations after discarding the first 50 periods, which approximates the long-time average. These plots demonstrate that the expectation value of the operator approaches, then oscillates about, a value that is very close (within a few percent) to the PGE prediction. Both the deviation of the average from the PGE prediction and the relative magnitude of the fluctuations about the mean value are shown to scale to zero with system size in Fig. 3.

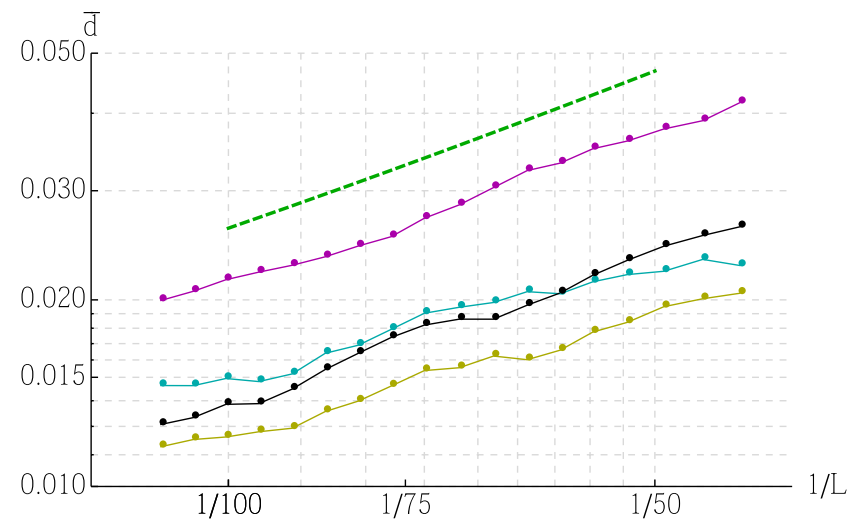

FIG. 3 (color online). Approach to equilibrium with system size. The Hamiltonian and color coding is the same as in Fig. 1. Here, $\bar{d}$ measures distance from the PGE prediction, $\bar{d}=(L N)^{-1} \sum_{m=n}^{n+N} \sum_{k}\left|\hat{n}(k, m T)-\hat{n}_{\mathrm{PGE}}(k)\right|$. We take $n=40 L$ and $N=20 L$, large enough so that the results are insensitive to further increase. The dashed green line is a plot of $\bar{d} \propto L^{-1}$ to guide the eye. These results strongly suggest that the distance of the long-time behavior of the system from our prediction at the thermodynamic limit falls off as a power law. prediction with increasing system size and (ii) to the steady state with time.

It is now natural to ask whether, and how, our results can be extended to a nonintegrable situation. Our PGE is analogous to the GGE for nondriven systems [5]; the analogy would suggest that, for a closed, nonintegrable, periodically driven system, a subsystem for which the rest of the system plays the role of a bath might be described by the periodic density matrix operator $\exp \left[\hat{H}_{\mathrm{eff}}(\epsilon)=\right.$ $\left.\hat{U}(0, \epsilon) \hat{H}_{\text {eff }} \hat{U}^{\dagger}(0, \epsilon)\right]$, analogous to the Gibbs ensemble for nondriven systems [11]. Unfortunately, there are several issues with this; chief amongst them are that $\hat{H}_{\text {eff }}(\epsilon)$ is not a local operator in general and, more seriously, that $\hat{H}_{\text {eff }}(\epsilon)$ is not uniquely defined (its eigenvalues are only defined modulo $2 \pi / T$ - we do not use the eigenvalues and, therefore, circumvent this problem in our work). We are currently investigating possible resolutions of these conceptual issues.

Our work here should be compared to the usual situation for out-of-equilibrium systems, where each case has to be studied individually using ad hoc techniques tailored to the specific problem at hand. In contrast, for this type of periodically driven system, the general framework of maximum entropy statistical mechanics applies as is. It not only gives the correct ensemble but also allows the detailed computation of physical observables. We hope that this work will motivate the search for further such "thermodynamic" principles governing driven systems in all generality.

We acknowledge discussions with $\mathrm{M}$. Aidelsburger, M. Atala, J. T. Barreiro, and I. Bloch. A. L. thanks M. Kollar, O. Tieleman, P. Ribeiro, A. Eckardt, T. Scheler, A. Sen, V. Bastidas, and M. Haque for discussions. A. D. acknowledges inspiring general discussions with E. Tosatti on nonequilibrium in the past.

[1] M. Srednicki, Phys. Rev. E 50, 888 (1994).

[2] J. M. Deutsch, Phys. Rev. A 43, 2046 (1991).

[3] M. Rigol, V. Dunjko, and M. Olshanii, Nature (London) 452, 854 (2008).

[4] M. A. Cazalilla, A. Iucci, and M.-C. Chung, Phys. Rev. E 85, 011133 (2012).

[5] M. Rigol, V. Dunjko, V. Yurovsky, and M. Olshanii, Phys. Rev. Lett. 98, 050405 (2007).

[6] P. Calabrese, F. H. L. Essler, and M. Fagotti, Phys. Rev. Lett. 106, 227203 (2011).

[7] P. Reimann, Phys. Rev. Lett. 101, 190403 (2008).

[8] M. Fagotti and F. H. L. Essler, Phys. Rev. B 87, 245107 (2013).

[9] E. T. Jaynes, Phys. Rev. 106, 620 (1957).

[10] S. Pressé, K. Ghosh, J. Lee, and K. A. Dill, Rev. Mod. Phys. 85, 1115 (2013).

[11] S. Popescu, A. J. Short, and A. Winter, Nat. Phys. 2, 754 (2006).

[12] J. H. Shirley, Phys. Rev. 138, B979 (1965). 
[13] H. Sambe, Phys. Rev. A 7, 2203 (1973).

[14] M. Grifoni and P. Hanggi, Phys. Rep. 304, 229 (1998).

[15] A. Das, Phys. Rev. B 82, 172402 (2010).

[16] A. Eckardt, C. Weiss, and M. Holthaus, Phys. Rev. Lett. 95, 260404 (2005).

[17] N. H. Lindner, G. Refael, and V. Galitski, Nat. Phys. 7, 490 (2011).

[18] T. Stöferle, H. Moritz, C. Schori, M. Köhl, and T. Esslinger, Phys. Rev. Lett. 92, 130403 (2004).

[19] H. Lignier, A. Zenesini, D. Ciampini, O. Morsch, E. Arimondo, S. Montangero, G. Pupillo, and R. Fazio, Phys. Rev. A 79, 041601 (2009).

[20] E. Haller, M. Gustavsson, M. J. Mark, J. G. Danzl, R. Hart, G. Pupillo, and H.-C. Naegerl, Science 325, 1224 (2009).

[21] T. Iadecola, C. Chamon, R. Jackiw, and S.-Y. Pi, Phys. Rev. B 88, 104302 (2013).

[22] Y.-A. Chen, S. Nascimbène, M. Aidelsburger, M. Atala, S. Trotzky, and I. Bloch, Phys. Rev. Lett. 107, 210405 (2011).

[23] A. Russomanno, A. Silva, and G. E. Santoro, Phys. Rev. Lett. 109, 257201 (2012).

[24] See Supplemental Material at http://link.aps.org/ supplemental/10.1103/PhysRevLett.112.150401 for details.
[25] M. Rigol and A. Muramatsu, Phys. Rev. A 70, 031603 (2004).

[26] M. Rigol and A. Muramatsu, Mod. Phys. Lett. B 19, 861 (2005).

[27] It is worth pointing out that $\hat{n}^{(b)}$ for the bosons is neither bilinear nor local in terms of the Jordan-Wigner fermions, since $\hat{b}_{i}^{\dagger} \hat{b}_{j}=\hat{a}_{i}^{\dagger}\left[\prod_{i \leq m<j}(-1)^{\hat{n}_{m}}\right] \hat{a}_{j}$. We, therefore, expect the PGE predictions to only approximate the real dynamics, becoming exact at the thermodynamic limit.

[28] A. Eckardt, M. Holthaus, H. Lignier, A. Zenesini, D. Ciampini, O. Morsch, and E. Arimondo, Phys. Rev. A 79, 013611 (2009).

[29] M. Atala, M. Aidelsburger, J. T. Barreiro, D. Abanin, T. Kitagawa, E. Demler, and I. Bloch, Nat. Phys. 9, 795 (2013).

[30] E. Haller, R. Hart, M. J. Mark, J. G. Danzl, L. Reichsöllner, M. Gustavsson, M. Dalmonte, G. Pupillo, and H. C. Nägerl, Nature (London) 466, 597 (2010).

[31] M. Olshanii, Phys. Rev. Lett. 81, 938 (1998).

[32] E. Haller, M. J. Mark, R. Hart, J. G. Danzl, L. Reichsöllner, V. Melezhik, P. Schmelcher, and H. C. Nägerl, Phys. Rev. Lett. 104, 153203 (2010). 